\title{
T7 peptide inhibits angiogenesis via downregulation of angiopoietin-2 and autophagy
}

\author{
FUHAI WANG $^{1}$, XIAOFENG DONG ${ }^{2}$, PENG XIU ${ }^{1}$, JINGTAO ZHONG $^{1}$, HONGLONG WEI $^{1}$, \\ ZONGZHEN XU ${ }^{1}$, TAO LI ${ }^{1}$, FENG LIU ${ }^{1}$, XUEYING SUN ${ }^{3}$ and $\mathrm{JIE} \mathrm{LI}^{1}$ \\ ${ }^{1}$ Department of General Surgery, Qianfoshan Hospital, Shandong University, Jinan, Shandong 250014; \\ ${ }^{2}$ Department of General Surgery, The People's Hospital of Guangxi Zhuang Autonomous Region, \\ Nanning 530021, P.R. China; ${ }^{3}$ Department of Molecular Medicine and Pathology, \\ Faculty of Medical and Health Sciences, University of Auckland, Auckland 1142, New Zealand
}

Received July 30, 2014; Accepted October 29, 2014

DOI: $10.3892 /$ or.2014.3653

\begin{abstract}
Angiogenesis is required for the invasion, metastasis and chemoresistance of tumor cells. In addition to vascular endothelial cell growth factor (VEGF), angiopoietin-2 (Ang2) is considered to be a promising target for anti-angiogenic therapy. The T7 peptide, an active fragment of full-length tumstatin [the noncollagenous 1 domain of the type IV collagen $\alpha 3$ chain, $\alpha 3$ (IV)NC1], has equivalent anti-angiogenic activity to that of full-length tumstatin. This study aimed to explore the mechanisms of the T7 peptide in the regulation of Ang2 expression in endothelial cells (ECs) as well as inhibition of angiogenesis and invasion of hepatocellular carcinoma cells. We also examined the role of autophagy in angiogenesis. Human umbilical vein endothelial cells (HUVECs) were incubated with endothelial cell medium (ECM)-2 in a hypoxia chamber to mimic hypoxic conditions. The recombinant $\mathrm{T} 7$ peptide inhibited the cell viability, tube formation and induced apoptosis of the HUVECs. The T7 peptide downregulated the protein expression of Ang2 by inhibiting phosphorylation of AKT under hypoxic conditions. The migration of HUVECs and invasion of HepG2 cells were inhibited by the T7 peptide via inhibition of Ang2 expression. EC autophagy was induced by the T7 peptide. Inhibition of autophagy enhanced the anti-angiogenic activity of the T7 peptide by increasing EC apoptosis. In vivo, immunohistochemistry of VE-cadherin and CD31 showed that angiogenesis was decreased significantly by the T7 peptide in a nude mouse xenogeneic tumor model. In conclusion, the T7 peptide inhibited angiogenesis and exerted its antitumor effects by inhibition of Ang2, phosphorylation of AKT and matrix metalloproteinase-2 (MMP-2) regulated by Ang2. Furthermore, inhibition of autophagy may significantly enhance the anti-angiogenic activity of the T7 peptide.
\end{abstract}

Correspondence to: Dr Jie Li, Department of General Surgery, Qianfoshan Hospital, Shandong University, 16766 Jingshi Road, Jinan, Shandong 250014, P.R. China

E-mail: 1ijie0503@126.com

Key words: angiogenesis, T7 peptide, AKT, apoptosis, migration, invasion, autophagy, angiopoietin-2

\section{Introduction}

Hepatocellular carcinoma (HCC) is a typical malignant tumor with an enriched blood supply. At the early stage of HCC, transarterial chemoembolization (TACE) is a treatment option, whereas anti-angiogenic drugs (such as sorafenib) are an option at the advanced stage of HCC $(1,2)$. Angiogenesis is a complicated process involving pro-angiogenic factors [e.g. vascular endothelial cell growth factor (VEGF), basic fibroblast growth factor, cyclooxygenase-2, angiopoietin-1 (Ang1)/Ang2)] and antiangiogenic factors (e.g. tumstatin, hexastatin and endostatin), which constitute the 'angiogenic switch' $(3,4)$. Tumor angiogenesis is closely associated with tumor growth, metastasis and chemoresistance. Recently, cytotoxic and anti-angiogenic drugs have been applied clinically and were found to improve the prognosis of HCC patients $(5,6)$. However, chemoresistance has attracted general attention and its definite mechanisms are being studied (7). Hypoxia induced by anti-angiogenic treatment activates hypoxia-inducible factor-1 $\alpha$ (HIF-1 $\alpha$ ) and nuclear factor- $\kappa \mathrm{B}(\mathrm{NF}-\kappa \mathrm{B})$ pathways (8). Indeed, hypoxia caused by anti-angiogenic drugs not only induces chemoresistance, but also results in local dissemination of tumor cells and distant metastasis (9). It has been suggested that sustained anti-angiogenic treatment may improve the poor prognosis of HCC patients to some extent. Nevertheless, it cannot be denied that high levels of Ang2 in HCC patient serum is an important marker of the poor treatment effect of sorafenib (10). Ang2 may be a potential factor in the modulation of angiogenesis, metastasis and chemoresistance of tumors under hypoxic conditions.

Ang2 is a member of the angiopoietin family which induces EC destabilization, disassociation from the endothelial cell medium (ECM), migration and sprouting (11). Ang2 is mainly stored in Weibel-Palade (WP) bodies of endothelial cells (ECs), which is released into the ECM by hypoxic stimulation, a hallmark of the intratumor environment. Ang2 and VEGF are considered as the main pro-angiogenic factors and Ang2 has been demonstrated to improve the pro-angiogenic activity of $\operatorname{VEGF}(12,13)$. Thus, Ang2 may be a key target molecule in future antitumor treatments.

Tumstatin is an endogenic anti-angiogenic factor derived from the noncollagenous 1 domain fragment of the type IV 
collagen $\alpha 3$ chain $(\alpha 3(\mathrm{IV}) \mathrm{NC} 1)$, which binds to integrin $\alpha \mathrm{V} \beta 3$ and $\alpha 3 \beta 1$ and further inhibits the activity of FAK/PI3K and NF-KB pathways under hypoxia (14). Downregulation of tumstatin is associated with enhanced cell transformation as well as tumor invasion and angiogenesis (15). The active fragments in full-length tumstatin are a current research focus. The T7 peptide, the 74-98 amino acid fragment of tumstatin, has an equivalent anti-angiogenic activity to that of full-length tumstatin (16). On the other hand, the T7 peptide exerts pro-apoptotic activity and inhibits the growth of tumors (17). However the anti-angiogenic mechanisms of the T7 peptide in tumor therapy are unclear. Tumstatin treatment of a diabetic nephropathy mouse model was found to decrease the expression of Ang-2, resulting in inhibition of glomerular hyper-filtration (18).

Autophagy is a process in which subcellular membranes undergo dynamic morphological changes that lead to degradation of cellular proteins and cytoplasmic organelles mediating the cell survival and metastatic ability of tumor cells $(19,20)$. Autophagy has been investigated as a mechanism of cell protection against drug-induced apoptosis in cancer cells by degradation of important proteins such as the pro-apoptotic protein Bax (21). Recently, it was reported that activation of autophagy induces pro-angiogenic activity under different contexts (22). High mobility group box 1 (HMGB1) was found to induce tube formation in vitro via induction of autophagy under hypoxic conditions (23). Moreover, the autophagic pathway crosstalks with the apoptotic pathway, which is considered to be a possible mechanism that modulates angiogenesis (24). However, the effect of the T7 peptide on autophagy and the role of autophagy in the T7 peptide-mediated inhibition of angiogenesis still remain unclear.

Anti-angiogenic therapy is important for the treatment of tumors, especially for tumors with an enriched blood supply. The related endogenic anti-angiogenic factors and pro-angiogenic factors have been examined in a previous study (3). The mechanisms of the $\mathrm{T} 7$ peptide in regulation of angiogenesis and invasion of HCC are still unknown. In the present study, we investigated the mechanisms of the T7 peptide in the inhibition of angiogenesis and whether the T7 peptide modulates the expression of Ang2 and the associated mechanisms. Moreover, we also examined the autophagy induced by the T7 peptide in modulation of angiogenesis. Our data provide evidence that Ang2, p-AKT, metalloproteinase-2 (MMP-2) and autophagy play important roles in angiogenesis and invasion of HCC, which can be directly or indirectly regulated by the T7 peptide.

\section{Materials and methods}

Cell lines and culture. Human umbilical vascular endothelial cells (HUVECs) were purchased from the Typical Animal Reserve Center of China (Shanghai, China) and cultured in ECM-2 medium (ScienCell Research Laboratories, Carlsbad, CA, USA) supplemented with $5 \%$ fetal bovine serum (FBS), $100 \mathrm{U} / \mathrm{ml}$ penicillin and $100 \mu \mathrm{g} / \mathrm{ml}$ streptomycin and $1 \%$ endothelial cell growth supplement (ECGS). The human HCC cell line HepG2 was obtained from the American Type Culture Collection (Rockville, MD, USA) and cultured in Dulbecco's modified Eagle's medium (DMEM) supplemented with $10 \%$ FBS, $100 \mathrm{U} / \mathrm{ml}$ penicillin and $100 \mu \mathrm{g} / \mathrm{ml}$ streptomycin. A hypoxia chamber (Billups-Rothenberg, Inc.) was used to mimic hypoxic conditions $\left(1 \% \mathrm{O}_{2}, 5 \% \mathrm{CO}_{2}\right.$ and $\left.94 \% \mathrm{~N}_{2}\right)$. Acetic acid $(30 \%)$ was used to adjust the $\mathrm{pH}$ of each group.

Antibodies and reagents. An antibody against Ang2 was purchased from Epitomics (Burlingame, CA, USA). Antibodies against CD31, Bcl-2, Bax, Akt, phosphorylated (p)-Akt (Ser473), MMP-2, light chain 3 (LC3) and GAPDH were purchased from Cell Signaling Technology (Danvers, MA, USA). An anti-VE-cadherin antibody was purchased from Abcam (Cambridge, UK). MK2206 was from Santa Cruz Biotechnology Inc. (Santa Cruz, CA, USA). Recombinant human Ang-2 (rhAng-2) was from PeproTech (Rocky Hill, NJ, USA). Recombinant T7 peptide was purchased from Bootech Bioscience and Technology (Shanghai, China) and dissolved in $30 \%$ acetic acid. Matrigel was purchased from BD Biosciences (San Jose, CA, USA).

Immunohistochemistry. HepG2 tumor samples from a xenogeneic nude mouse model (following the approval of the Ethics Committee of Qianfoshan Hospital) were fixed in 10\% formalin, embedded in paraffin and then processed for immunohistochemistry. Sections were deparaffinized in graded xylene and rehydrated in graded ethanol, followed by 3 washes with phosphate-buffered saline (PBS) for 3 min each. After heat-induced antigen retrieval in citrate buffer, endogenous peroxidase was inhibited by treatment with $3 \%$ hydrogen peroxide at room temperature for $10 \mathrm{~min}$, followed by 3 washes with PBS for 3 min each. Primary anti-VE cadherin (1:50 dilution) and antiCD31 (1:30 dilution) antibodies were applied overnight. After washing, the sections were incubated with horseradish peroxidase (HRP)-conjugated goat anti-rabbit IgG (1:50 dilution; Beyotime, Jiangsu, China) for $1 \mathrm{~h}$ at $37^{\circ} \mathrm{C}$. Negative control sections were incubated with PBS instead of the primary antibody.

Tube formation assay. HUVECs $\left(5 \times 10^{3} /\right.$ well) were seeded in 96-well plates coated with Matrigel and allowed to adhere. The cells were then incubated under normoxic or hypoxic conditions with or without the T7 peptide and/or 3-MA (5 nM) for $24 \mathrm{~h}$ at $37^{\circ} \mathrm{C}$ with $5 \% \mathrm{CO}_{2}$.

Cell viability assay. HUVECs $\left(5 \times 10^{3} /\right.$ well $)$ were seeded in two 96-well plates. After $12 \mathrm{~h}$, the medium in the first plate was replaced with medium containing various concentrations of the T7 peptide $(0.25,0.5,1$ and $2 \mu \mathrm{M})$ and then the cells were incubated in the hypoxia chamber. HUVECs in the second plate were incubated under hypoxic conditions with or without the $\mathrm{T} 7$ peptide $(1 \mu \mathrm{M})$. Control and treatment groups were adjusted to the same $\mathrm{pH}$ with $30 \%$ acetic acid. At various time-points (plate 1: $\mathrm{t}=24 \mathrm{~h}$; plate 2: $\mathrm{t}=12$ and $24 \mathrm{~h}$ ), the cells were applied to a Cell Counting Kit-8 (Dojindo Molecular Technologies, Japan). The optical density (OD) at $450 \mathrm{~nm}$ was measured with a Spectra Max 190 (Molecular Devices, Sunnyvale, CA, USA).

Cell apoptosis assay. HUVECs ( $4 \times 10^{5} /$ well) were seeded in a 6-well plate. After $12 \mathrm{~h}$, the cells were incubated with fresh medium under normoxic or hypoxic conditions with or without the T7 peptide and/or 3-MA $(5 \mu \mathrm{M})$. After $24 \mathrm{~h}$, the cells were trypsinized and centrifuged at $1,000 \mathrm{x}$ g for $10 \mathrm{~min}$. Then, the 
A

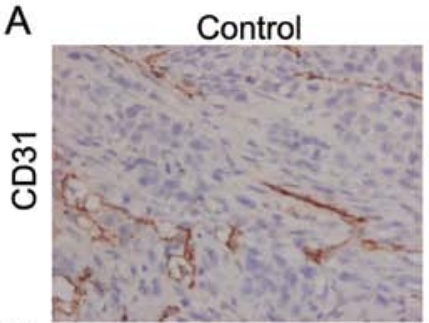

C

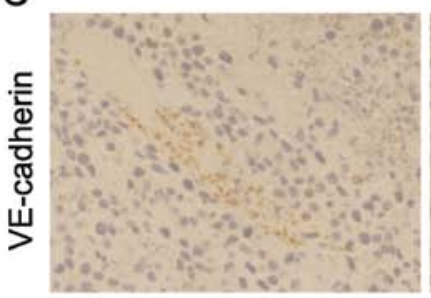

E

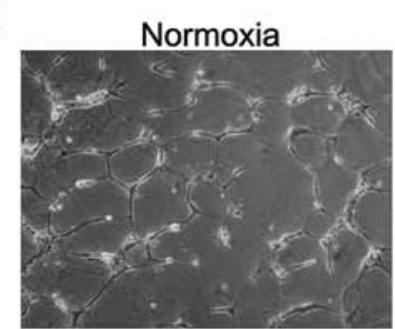

T7 peptide
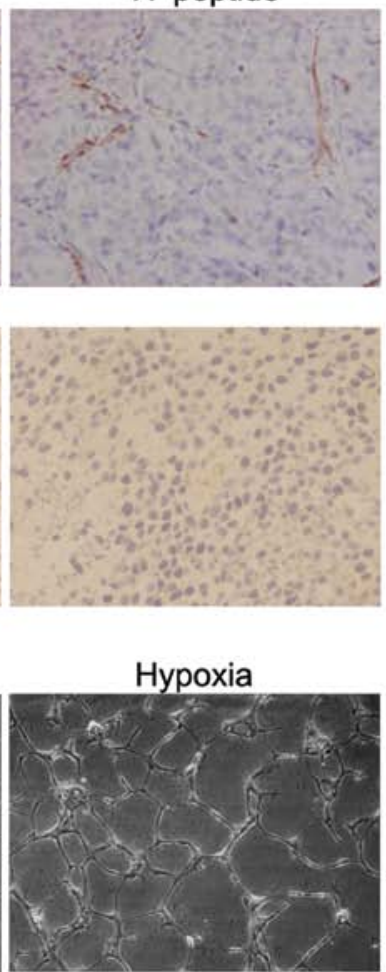

B
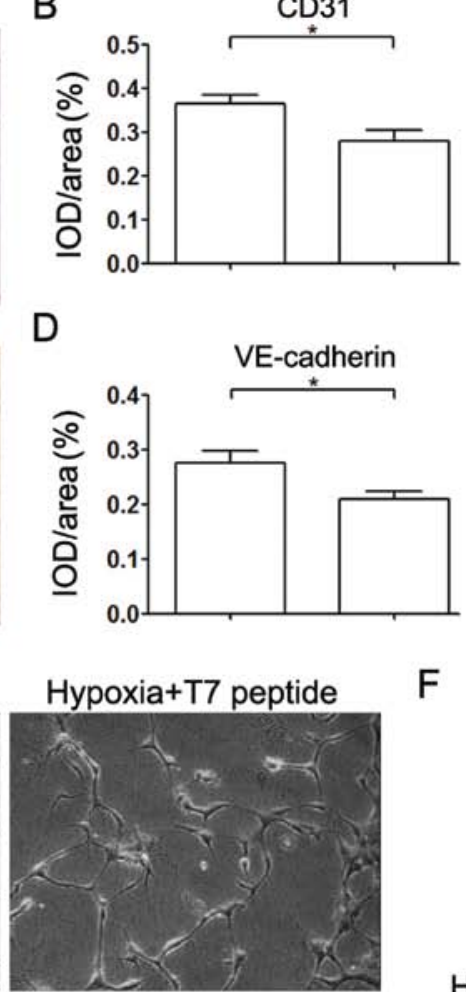

F

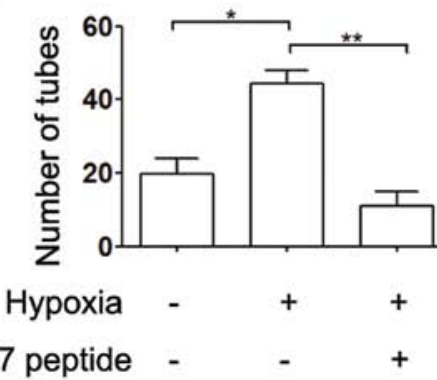

Figure 1. T7 peptide decreases vessel formation in vivo and tube formation in vitro. (A and C) Two molecular markers of angiogenesis in tumors, CD31 and VE-cadherin, were stained in the control group and treatment group, respectively (magnification, x400). (B and D) The mean density (IOD/area) was counted with Image-Pro Plus 6.0. (E) Human umbilical vein endothelial cells (HUVECs) were incubated for the tube formation assay under normoxic condition or hypoxic condition with or without the T7 peptide (magnification, $\mathrm{x} 200$ ). (F) The average number of tubes in the different groups was obtained from 3 independent views. ${ }^{*} \mathrm{P}<0.05,{ }^{* *} \mathrm{P}<0.01$

cells were washed twice with sterile PBS and resuspended with binding buffer. Annexin V/FITC and propidium iodide (PI; NeoBioscience Ltd.) were used to detect the apoptosis rate of cells according to the manufacturer's instructions. Finally, Annexin V-and PI-labeled cells were analyzed by flow cytometry.

Western blot analysis. At $80 \%$ confluency, HUVECs were incubated under normoxic or hypoxic conditions with or without the T7 peptide $(1 \mu \mathrm{M})$ or MK2206 $(5 \mu \mathrm{M})$ and/or 3-MA (5 nM), respectively. HepG2 cells were incubated with the supernatants from the HUVECs divided into 4 groups: normoxic group, hypoxic groups with or without the T7 peptide plus rhAng2 $(15 \mathrm{ng} / \mathrm{ml})$ or not. After $24 \mathrm{~h}$, the cells were lysed in cold RIPA lysis buffer (Beyotime) with $1 \mathrm{nM}$ phenylmethylsufonyl fluoride, followed by centrifugation at $12,000 \mathrm{x} \mathrm{g}$ for $10 \mathrm{~min}$ at $4^{\circ} \mathrm{C}$. The concentration of the protein samples was measured with a BCA Protein Assay kit (Beyotime). Total proteins (20-40 $\mu \mathrm{g}$ ) were separated on 8-15\% SDS-polyacrylamide gel electrophoresis gels (Beyotime) and electrotransferred to polyvinylidene fluoride membranes. The membranes were then incubated with primary antibodies overnight at $4^{\circ} \mathrm{C}$ [1:3,000 for Ang2; 1:1,000 for Bcl-2, Bax, Akt, p-Akt (Ser473), MMP-2 and LC3]. After 3 washes with PBS containing $0.1 \%$ Tween-20 for $15 \mathrm{~min}$ each, the membranes were incubated with HRP-conjugated anti-rabbit $\mathrm{IgG}$ (Beyotime) at $37^{\circ} \mathrm{C}$ for $1 \mathrm{~h}$ and then washed 3 times with
PBS containing $0.1 \%$ Tween-20. A TANON-4500SF chemiluminescence system was used to detect the target proteins.

Migration assay. HUVECs were seeded in 24-well plates with ECM-2 medium. At confluency, a wound was induced by scratching the monolayer with a $10-\mu 1$ pipette tip. The cells were then washed 3 times with sterile PBS. HUVECs were incubated in serum-free ECM-2 medium under normoxic or hypoxic conditions with or without the T7 peptide $(1 \mu \mathrm{M})$ plus rhAng2 $(15 \mathrm{ng} / \mathrm{ml})$ or not. Images were acquired at $12 \mathrm{~h}$ post-scratching.

HUVECs ( $1 \times 10^{5}$ in $200 \mu 1$ medium) were added to the upper chamber of a Transwell plate, and $500 \mu 1$ medium as described for the scratch assay was added to the lower chamber. After $24 \mathrm{~h}$, the migrated cells were fixed with 95\% methanol and stained with $0.1 \%$ crystal violet for $30 \mathrm{~min}$ followed by 5 washes with PBS.

Invasion assay of HepG2. HUVECs (2×105/well) were seeded in the lower chamber of a Transwell plate, and HepG2 cells (1x10 $/$ well) were added to the upper chamber coated with Matrigel. After $12 \mathrm{~h}$, the culture medium for the HUVECs was replaced and the cells were incubated under normoxic or hypoxic conditions with or without the T7 peptide $(1 \mu \mathrm{M})$ plus rhAng-2 $(15 \mathrm{ng} / \mathrm{ml})$ or not. After $48 \mathrm{~h}$, HepG2 cells in the upper chamber were fixed with $95 \%$ methanol and stained with $0.1 \%$ crystal violet for $30 \mathrm{~min}$ followed by 5 washes with PBS. 
A

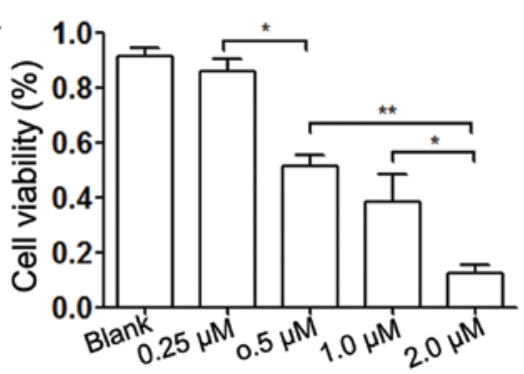

B

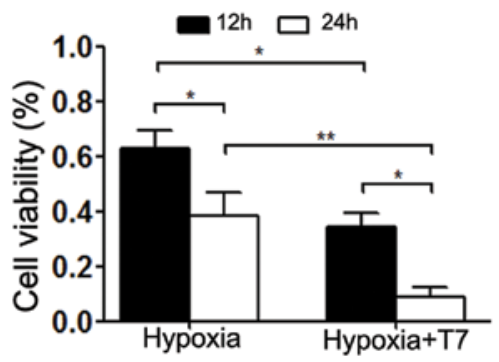

C
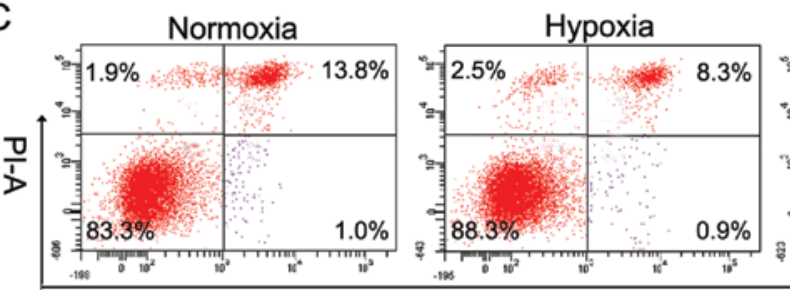

Hypoxia+T7 peptide

D

FITC-A
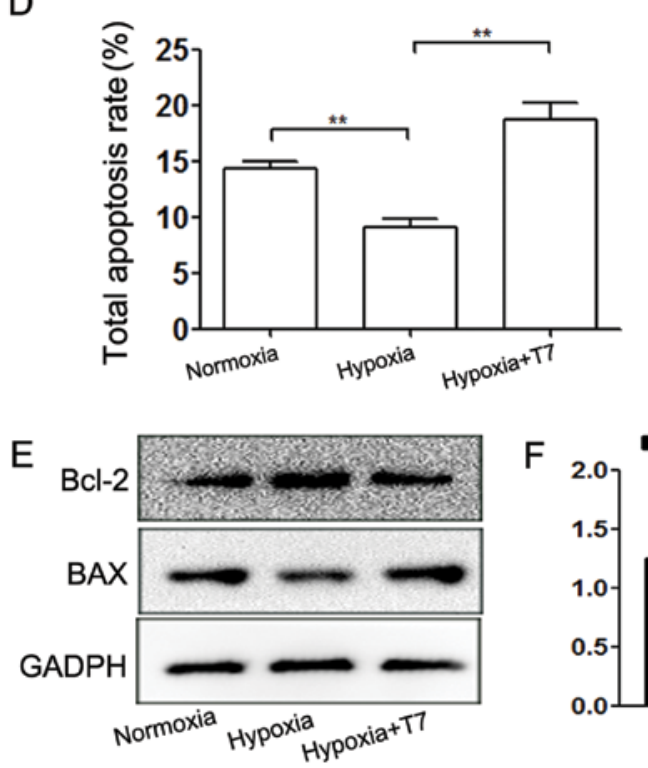

$F$

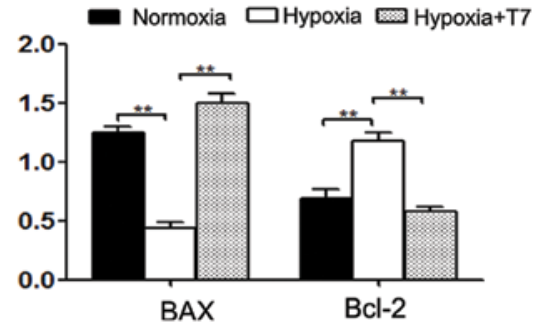

Figure 2.T7 peptide inhibits cell viability and induces apoptosis of human umbilical vein endothelial cells (HUVECs) under hypoxic conditions. (A) HUVECs were incubated under hypoxic conditions with different concentrations of the T7 peptide for $24 \mathrm{~h}$, respectively. (B) HUVECs were incubated under hypoxic condition with or without the T7 peptide $(1.0 \mu \mathrm{M})$ for the cell viability assay. (C) HUVECs were incubated under normoxic or hypoxic conditions with or without the $\mathrm{T} 7$ peptide for $24 \mathrm{~h}$ for the apoptosis assay. (D) The average total apoptosis rate was obtained from 3 independent experiments. (E) Expression of Bax and Bcl-2 was analyzed by western blotting analysis. (F) The density of each band was quantified and normalized to that of GADPH. " $\mathrm{P}<0.05$, ${ }^{* *} \mathrm{P}<0.01$.

Enzyme-linked immunosorbent assay (ELISA). HUVECs were seeded in 6-well plates and incubated overnight. Then, the medium was changed and the HUVECs were incubated under normoxic or hypoxic conditions with or without the T7 peptide. After $24 \mathrm{~h}$, the culture supernatants were collected. Measurement of Ang2 levels in the culture supernatants was performed using a commercially available ELISA kit (Gr Bio, Shanghai, China) according to the manufacturer's instructions. OD values were obtained at $450 \mathrm{~nm}$ by the Spectra Max 190.

Acridine orange staining for cell autophagy. HUVECs (5x $10^{3} /$ well) were seeded in 24-well plates with ECM-2 medium. After $6 \mathrm{~h}$, the cells were incubated with new medium under normoxic or hypoxic conditions with or without T7 peptide $(1 \mu \mathrm{M})$ and/or 3-MA $(5 \mathrm{nM})$ for $24 \mathrm{~h}$. The cells were then stained with an acridine orange solution $(5 \mu \mathrm{M})$ for $10 \mathrm{~min}$ at $37^{\circ} \mathrm{C}$ and then washed 3 times with PBS. The cells were observed and photographed under a fluorescence microscope.

Statistical analysis. The Student's t-test was used for comparisons between 2 groups or one-way analysis of variance for comparisons between multiple groups. Statistical analysis was performed with SPSS software (version 17.0, SPSS China, Shanghai, China). $\mathrm{P}<0.05$ was considered to indicate a statistically significant result.

\section{Results}

$T 7$ peptide inhibits angiogenesis in vivo and in vitro. First, we examined the expression levels of CD31 and VE-cadherin, which are hallmarks of angiogenesis, by immunohistochemical analysis. The expression of both CD31 and VE-cadherin was 


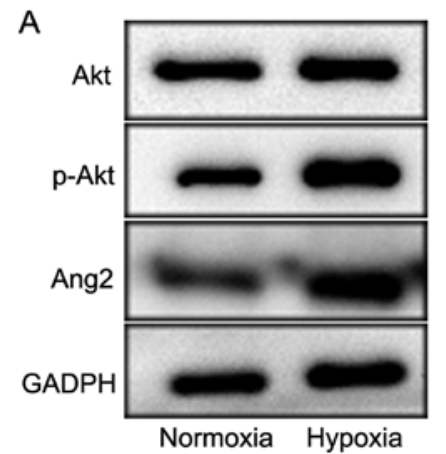

C

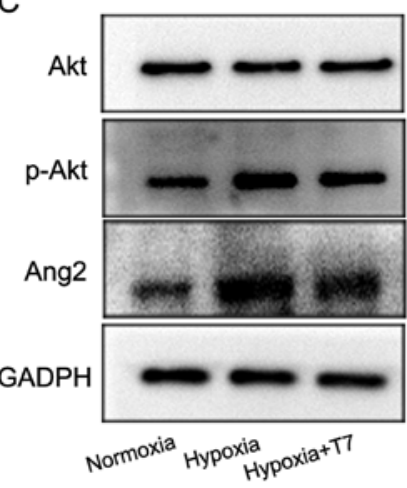

$\mathrm{E}$

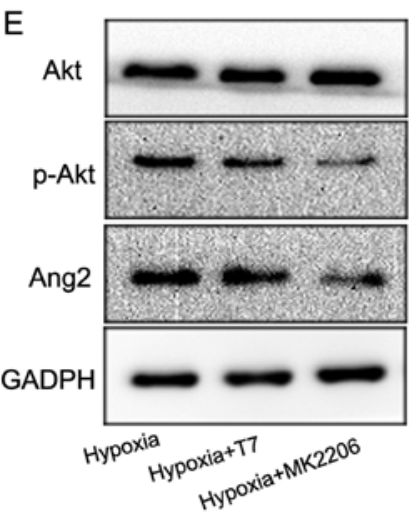

B

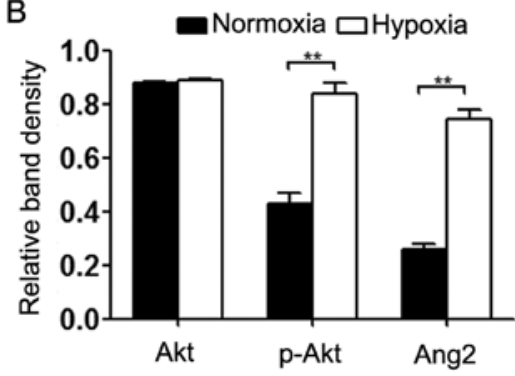

D

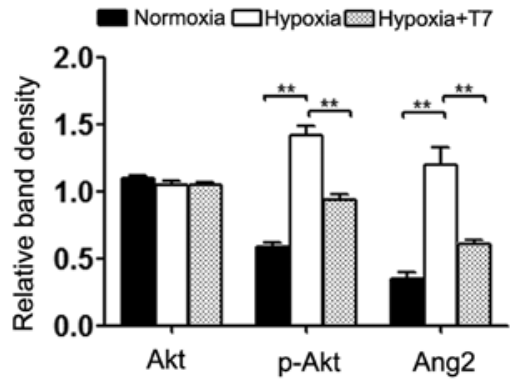

$\mathrm{F}$

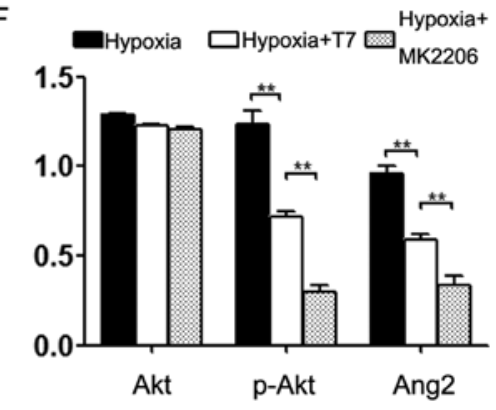

Figure 3. T7 peptide inhibits expression of Ang2 via inhibition of the Akt pathway. (A) Expression of Akt, p-Akt and Ang2 under normoxic or hypoxic conditions was detected by immunoblotting. (C) Expression of Akt, p-Akt and Ang2 under normoxic or hypoxic conditions with or without the T7 peptide was detected by immunoblotting. (E) Expression of Akt, p-Akt and Ang2 under hypoxic condition with the T7 peptide or 3-MA was also detected by immunoblotting. (B, D and F) The density of each band was quantified and normalized to that of GADPH. ${ }^{*} \mathrm{P}<0.05,{ }^{* *} \mathrm{P}<0.01$.

downregulated in the $\mathrm{T} 7$ peptide treatment group and angiogenesis was decreased compared with that in the control group (Fig. 1A-D). To further investigate the effect of the T7 peptide on angiogenesis, we incubated HUVECs in Matrigel-coated 96-well plates under various conditions for $24 \mathrm{~h}$. The results indicated that capillary-like tube formation was decreased significantly by the T7 peptide under hypoxic conditions in vitro, which was consistent with the in vivo results (Fig. $1 \mathrm{E}$ and F).

T7 peptide inhibits cell viability and induces apoptosis under hypoxic conditions. Under hypoxic conditions, the T7 peptide contributed to a significant decrease in cell viability in both dose- and time-dependent manners (Fig. 2A and B). We further detected the degree of apoptosis caused by the T7 peptide under hypoxic conditions, which is one of the factors affecting cell viability. The results revealed that the T7 peptide enhanced the apoptosis rate significantly, whereas hypoxia suppressed apoptosis (Fig. 2C and D). Western blot analysis indicated that the $\mathrm{T} 7$ peptide reduced the expression of the anti-apoptotic protein Bcl-2 and reversed the decrease in the expression of pro-apoptotic protein Bax induced by hypoxia (Fig. 2E and F).

T7 peptide decreases Ang2 expression via inhibition of AKT phosphorylation in ECs. Ang2 is crucial for disassociation of ECs from the ECM, EC migration and sprouting and the induction of angiogenesis in HCC (25). To explore the mechanism of the $\mathrm{T} 7$ peptide in the modulation of Ang2 expression, we first detected the expression levels of Akt, p-Akt and Ang2 under hypoxic conditions. Hypoxia significantly increased the expression of Ang2, which was accompanied by Akt activation (Fig. 3A and B). Based on these results, we treated the cells with the T7 peptide under hypoxic conditions. As a result, the Ang2 expression level was downregulated significantly by the T7 peptide when the Akt pathway was inhibited 

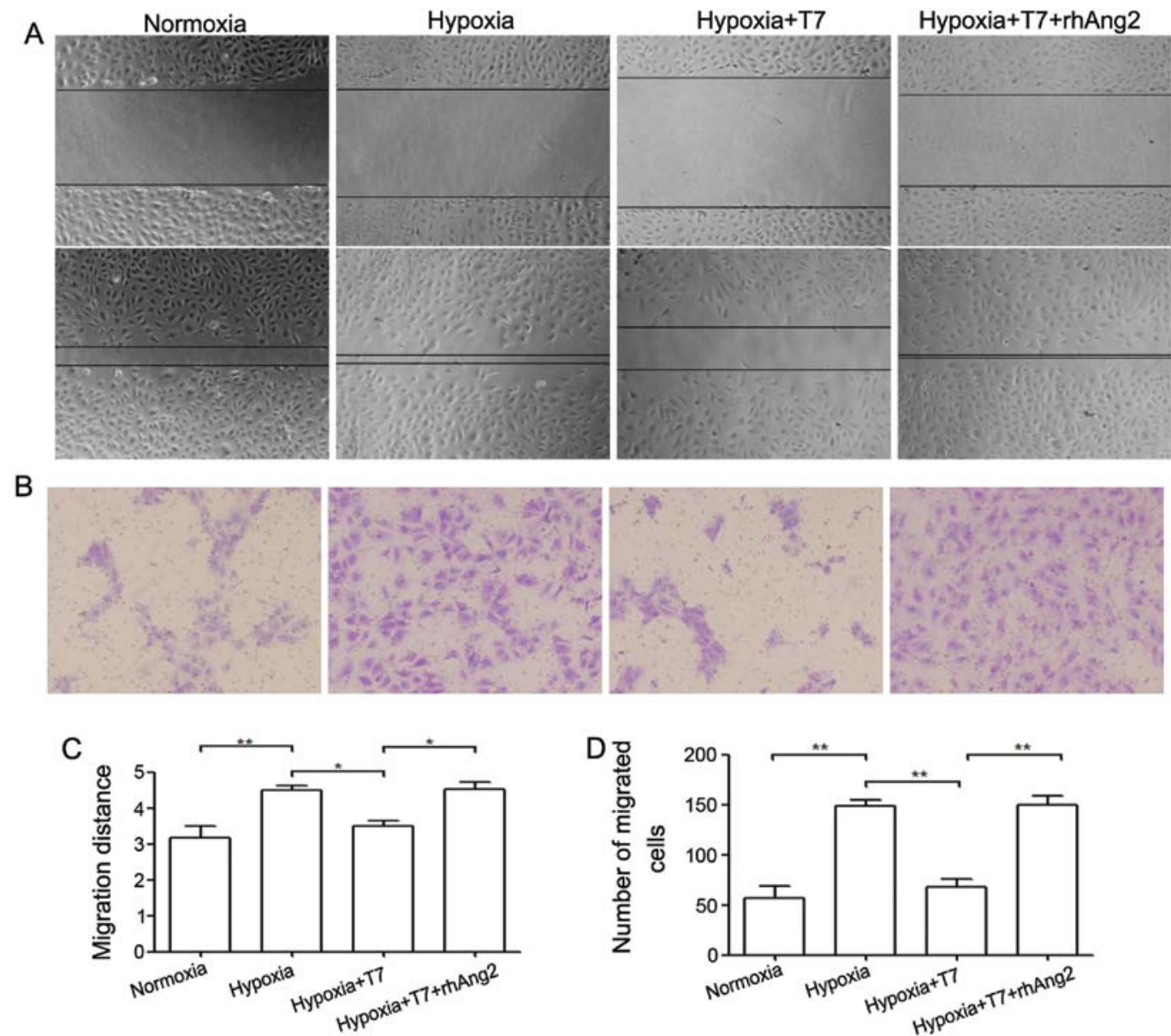

Figure 4. T7 peptide inhibits the migration of human umbilical vein endothelial cells (HUVECs) via inhibition of Ang2. (A) The effects of the T7 peptide and rhAng2 on the migration of HUVECs were analyzed by a scratch assay (magnification, x100). (B) Transwell plates were used to repeat the migration assay grouped in the same manner as the scratch assay (magnification, $\mathrm{x} 100$ ). (C) Migration distances were identified as the average migration distance of 5 equidistance spots. (D) The average number of migrated cells was obtained from 5 random views. ${ }^{*} \mathrm{P}<0.05,{ }^{* *} \mathrm{P}<0.01$.

simultaneously, while the amount of total Akt was unchanged (Fig. 3C and D). To examine the relationship between Ang2 expression and the Akt pathway, we incubated HUVECs under hypoxic conditions with the T7 peptide or MK2206 (positive control) for $24 \mathrm{~h}$. Western blotting showed that MK2206 significantly reduced the protein levels of Ang2 and p-Akt, which was consistent with the results of the T7 peptide treatment (Fig. 3E and F). Based on these data, blocking the phosphorylation of Akt contributed to the downregulation of Ang2. Furthermore, the T7 peptide reduced the expression of Ang2 via inhibition of Akt phosphorylation.

T7 peptide reduces EC migration via downregulation of Ang2. The process of angiogenesis is initiated by quiescent ECs that become activated by angiogenic signals. Angiogenesis proceeds through vessel destabilization, EC migration, proliferation and sprouting and coverage of pericytes and muscle cells (26). Ang2 plays a key role in modulation of EC disassociation and migration (27). To determine whether the T7 peptide inhibits EC migration, we first analyzed HUVECs in a scratch assay. Since we demonstrated that the T7 peptide reduced Ang2 expression in a previous study, we treated the cells with the T7 peptide and rhAng2. We found that the migration ability of HUVECs was enhanced by hypoxia compared with that under normoxia, whereas the $\mathrm{T} 7$ peptide reduced cell migration ability significantly. Our data also showed that rhAng2 significantly reversed the inhibition of the $\mathrm{T} 7$ peptide under hypoxic condition (Fig. 4A and C). To confirm these results, we applied HUVECs to a migration assay and the results were consistent with those of the scratch assay (Fig. 4B and D). Based on these data, the T7 peptide inhibited EC migration by a reduction in the protein level of Ang2, leading to inhibition of angiogenesis.

Ang2 mediates the invasion of HCC cells. Ang2 plays an important role not only in the angiogenesis of $\mathrm{HCC}$, but also in the metastasis of HCC (24). We measured the concentration of Ang2 in culture supernatants of HUVECs by ELISA (Fig. 5C). Hypoxia induced Ang2 release from WP bodies and the T7 peptide blocked this process significantly. To explore the role of Ang2 in modulation of the invasive ability of HepG2 cells, we incubated HepG2 cells for $24 \mathrm{~h}$ in the upper chamber of a Transwell plate with HUVECs seeded in the lower chamber. Rapid release of Ang2 induced by hypoxia enhanced the invasive ability of HepG2 cells, whereas the T7 peptide abrogated HepG2 cell invasion by downregulating the expression of Ang2 (Fig. 5A and B). Moreover, addition of rhAng2 to the T7 peptide treatment group confirmed that Ang2 played a key role 


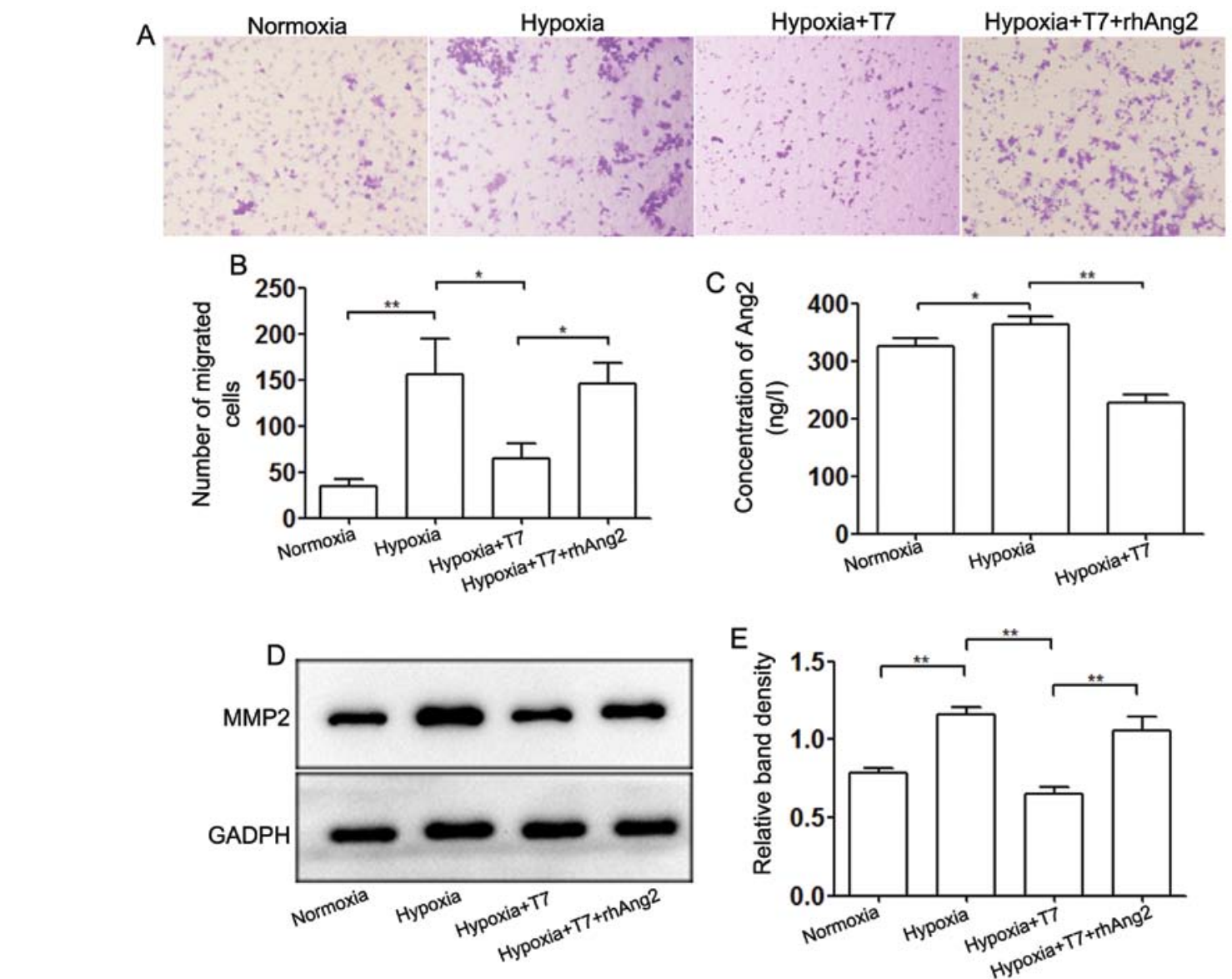

Hypoxia+T7 Hypoxia+T7+rhAng2

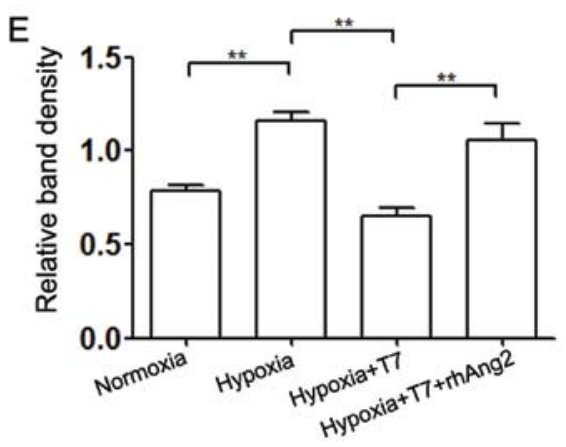

Figure 5. T7 peptide inhibits the invasion of HepG2 cells by inhibition of Ang2 expression. (A) Transwell plates were used to analyze the effect of the T7 peptide and Ang2 on the invasion of HepG2 cells (magnification, x100). (B) The number of migrated cells was counted from 5 random views. (C) ELISA kit was used to detect the concentrations of Ang2 in supernatants of the human umbilical vein endothelial cell (HUVEC) culture. (D) Expression of MMP-2 was analyzed by western blotting. (E) The density of each band was quantified and normalized to that of GADPH. ${ }^{*} \mathrm{P}<0.05,{ }^{* *} \mathrm{P}<0.01$.

in mediating tumor cell invasion regulated by the $\mathrm{T} 7$ peptide and hypoxia. We also detected the expression level of MMP-2, which is related to tumor metastasis. The data showed that downregulation of Ang2 induced by the T7 peptide decreased the expression level of MMP-2 under hypoxic conditions, whereas rhAng2 partially neutralized the inhibition (Fig. 5D and $\mathrm{E}$ ). These results indicated that the $\mathrm{T} 7$ peptide inhibited the invasion of HepG2 cell by reducing expression of MMP-2, which was mediated through Ang2.

Inhibition of autophagy potentiates the anti-angiogenic activity of the $T 7$ peptide. The T7 peptide inhibited capillarylike tube formation by inhibition of EC viability, migration mediated by Ang2 and induction of EC apoptosis. Previous studies have shown that autophagy plays a dual role in regulating angiogenesis $(22,28)$. In our study, we incubated ECs under hypoxic conditions and found that the T7 peptide induced autophagy compared with hypoxia (Fig. 6A). LC3-II is a marker protein of the level of autophagy (29). The results of our western blot analysis of LC3-II were in accordance with the data from acridine orange (AO) staining (Fig. 6B and $\mathrm{C}$ ). To further examine the role of autophagy in angiogenesis, we incubated ECs under hypoxic conditions in a tube formation assay followed by OA staining. The level of autophagy induced by the T7 peptide in HUVECs was significantly downregulated by 3-MA, a specific inhibitor of autophagy (Fig. 6D-F). Furthermore, 3-MA inhibited tube formation under hypoxic conditions, and the anti-angiogenic ability of the T7 peptide was enhanced by addition of 3-MA (Fig. 7A and $\mathrm{C}$ ). An apoptosis assay indicated that 3-MA increased the pro-apoptotic activity of the T7 peptide (Fig. 7B and D). However, the effect of the T7 peptide was more obvious than that of 3-MA (Fig. 7A and B). Based on these data, we demonstrated that the $\mathrm{T} 7$ peptide reduced the tube formation in vitro by induction of EC apoptosis and inhibition of autophagy. 3-MA synergistically enhanced the anti-angiogenic ability of the T7 peptide. Autophagy may be a protective mechanism against the anti-angiogenic activity of the T7 peptide.

\section{Discussion}

Angiogenesis-targeted treatment is one of the most appropriate therapeutic options for HCC patients, especially for advanced patients (1).Tumor angiogenesis mostly depends on the balance between pro-angiogenic and anti-angiogenic factors $(3,4)$. Hypoxia, which is particularly present inside tumors, induces the expression of pro-angiogenic factors such as VEGF and Ang2 (30). Anti-angiogenic treatment in HCC patients is considered to be a factor that induces intratumor hypoxia (8). The T7 peptide, an active fragment 
A

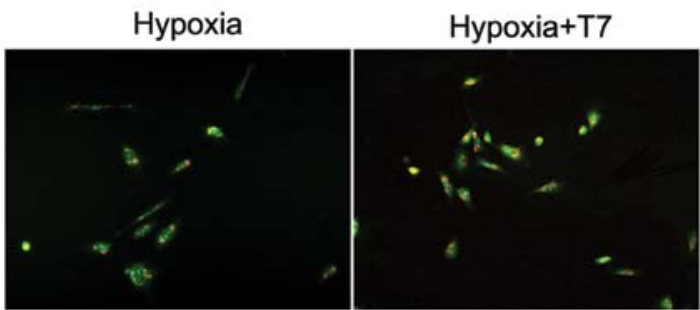

B

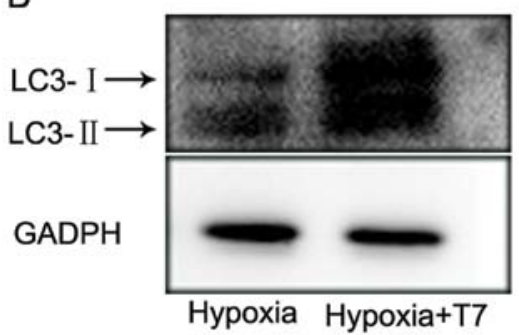

C

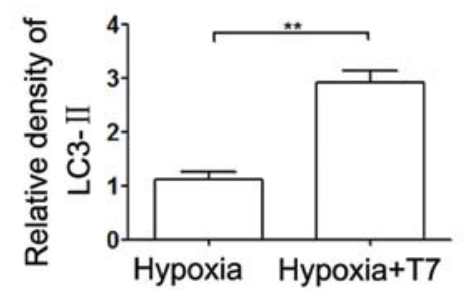

D
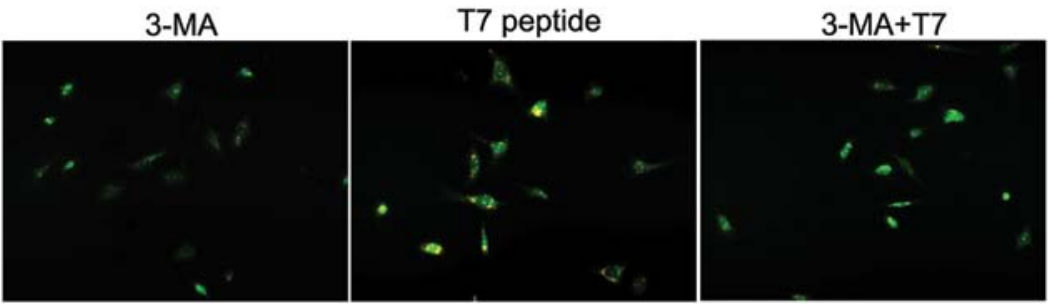

E
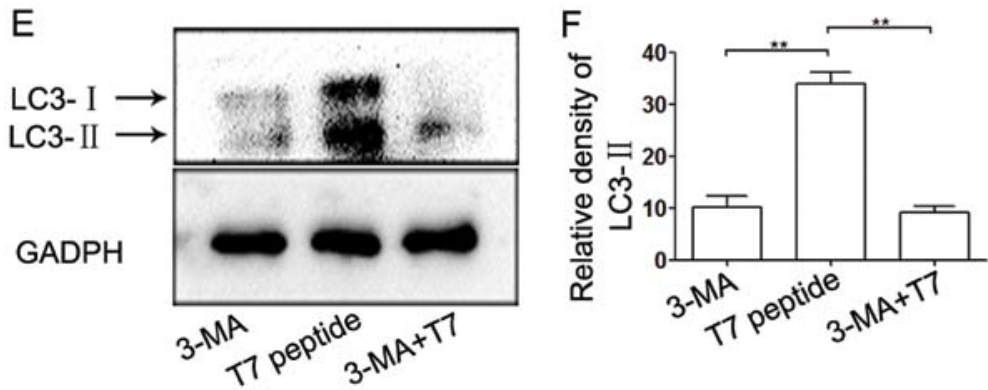

Figure 6. T7 peptide induces autophagy of human umbilical vein endothelial cells (HUVECs). (A and D) Acridine orange was used to stain HUVECs for detecting the level of autophagy (magnification, $\mathrm{x} 400$ ). (B and E) LC3-I/II were identified by immunoblotting. (C and F) The density of each band was quantified and normalized to that of GADPH. ${ }^{* *} \mathrm{P}<0.01$.

of full-length tumstatin, exerts an anti-angiogenic activity through its L, V and D amino acids (31). Compared with full-length tumstatin, the T7 peptide owns lower molecular weight and better commercial availability. Therefore, the T7 peptide may be a better prospect for the anti-angiogenic therapy of HCC patients. Our data showed that the T7 peptide inhibited angiogenesis in vivo. CD31 is considered as a marker that indicates the degree of tumor angiogenesis. VE-cadherin is a key component of endothelial cell-to-cell adherens junctions and plays an important role in vascular permeability and remodeling. VE-cadherin is required for organization of a stable vascular system and controls vascular permeability in adults (32). Immunohistochemical staining of CD31 and VE-cadherin indicated that treatment with the T7 peptide resulted in reduced vessel formation. Moreover, the present study also indicated that the $\mathrm{T} 7$ peptide exerts its anti-angiogenic ability through inhibiting EC viability and migration and inducing apoptosis in vitro. The level of Ang2 is rapidly elevated in tumor-associated endothelium (33), which promotes EC disassociation from the ECM and migration. Blocking the Ang2/Tie2 axis inhibits tumor angiogenesis, growth and dissemination in multiple tumor models, including tumors that are prone to developing resistance against anti-VEGF/VEGF receptor therapy (34). A previous study reported that AMG386 (an Fc fusion peptide that blocks the Ang-2/Tie2 axis) shows antitumor activity in patients with clear cell metastatic renal cell carcinoma (35). Ang2 blockade also reduces tumor vessel sprouting, and ectopic high expression of Ang2 inhibits the benefits of anti-VEGF receptor treatment $(36,37)$. Thus, a high Ang2 level is not only responsible for the initiation of angiogenesis but also is partially responsible for chemoresistance against sorafenib, suggesting that Ang2, as a target of anti-angiogenic treatment, should be investigated further. The present study demonstrated that the T7 peptide significantly decreased the expression of Ang2 under hypoxic conditions, which was significantly elevated by hypoxia. Akt promotes tumorigenesis and drug resistance by disrupting apoptosis (38). Akt phosphorylation was induced by hypoxia, which indicates activation of the Akt pathway, whereas the T7 peptide inhibited phosphorylation of Akt. 
A
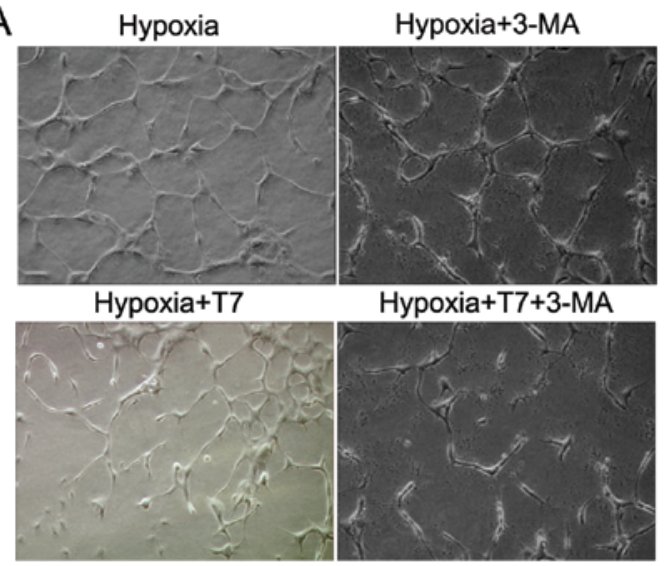

C

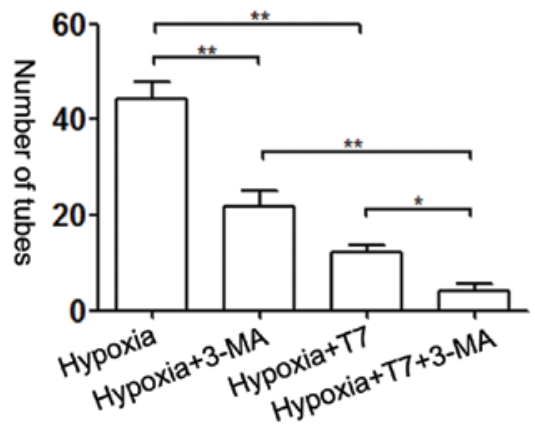

B
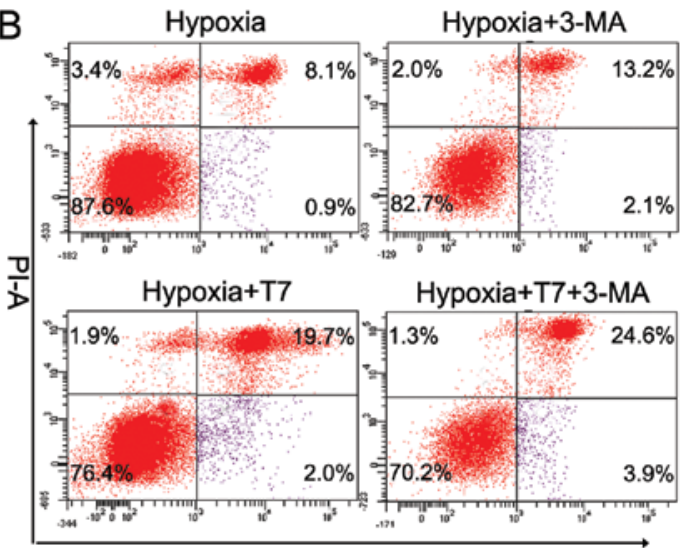

FITC-A

D

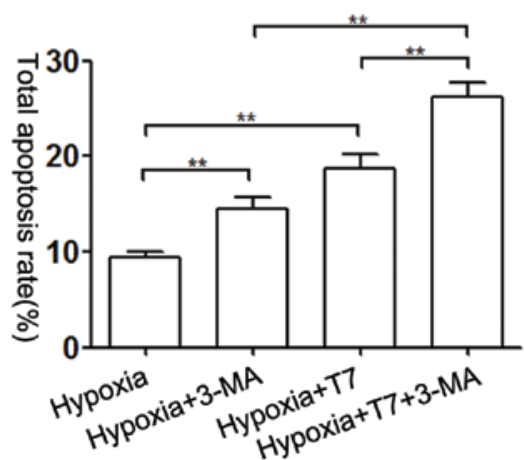

Figure 7. Inhibition of autophagy enhances the anti-angiogenic ability of the T7 peptide. (A) Human umbilical vein endothelial cells (HUVECs) were incubated under hypoxic conditions with the T7 peptide and/or 3-MA for the tube formation assay (magnification, x200). (B) Effect of the T7 peptide and 3-MA on the apoptosis of HUVECs was detected. (C) The average number of tubes in the different groups was obtained from 3 independent views. (D) The average total apoptosis rate was obtained from 3 independent experiments. ${ }^{*} \mathrm{P}<0.05,{ }^{* *} \mathrm{P}<0.01$.

MK2206, an inhibitor of the Akt pathway, also significantly downregulated the Ang2 expression level, which was accompanied by inhibition of Akt phosphorylation. We conclude that the T7 peptide reduced Ang2 expression by inhibiting phosphorylation of Akt.

Angiogenesis involves EC dissociation, migration, sprouting, vessel formation and coverage of pericytes. EC migration is indispensable for angiogenesis (26). Our data showed that the T7 peptide inhibited hypoxia-mediated cell migration and addition of rhAng 2 mostly neutralized the effect of the T7 peptide. EC migration was inhibited by the T7 peptide by downregulating Ang2 expression. It has been reported that overexpression of Ang2 enhances tumor metastasis and an Ang2-blocking antibody inhibits this process (39). MMP-2 is responsible for degradation of the ECM. High expression of MMP-2 is important for invasion of tumor cells (25). Consistent with previous studies, our study demonstrated that the invasive ability of HepG2 cells was increased by hypoxia with high expression of MMP-2, whereas the T7 peptide inhibited these effects. The high expression of Ang2 under hypoxic conditions may be related to the invasion of HepG2 cells and the mechanisms of the T7 peptide in the inhibition of HepG2 cell invasion may involve downregulation of Ang2. Moreover, the addition of rhAng2 partly reversed the inhibitory activity of the $\mathrm{T} 7$ peptide, which verifies our hypothesis. These results indicate that the anti-metastatic ability of the T7 peptide was mediated by partially inhibiting Ang2. However, the underlying mechanism of Ang2 in the enhancement of tumor cell invasion still needs to be explored.

Autophagy is a self-catabolic cellular process in which cytoplasmic proteins and organelles are sequestered and delivered to lysosomes for degradation (40). In most cases, autophagy is considered as a cell survival mechanism induced under certain conditions of stress. A previous study reported that elevated autophagy of ECs improves capillary-like tube formation in vitro by potentiating the anti-apoptotic ability (22). Interestingly, it has also been reported that autophagy increases angiogenesis by enhancing the proliferation of ECs (28). Therefore, autophagy has been suggested to play an adverse role in angiogenesis under different contexts. Our present study indicated that the T7 peptide induced autophagy of HUVECs and upregulated the expression of LC3-II. 3-MA, a specific inhibitor of autophagy, inhibited the angiogenesis. Compared with the T7 peptide, 3-MA had a lesser effect on the tube formation and apoptosis of ECs. Meanwhile, 3-MA enhanced the pro-apoptotic and anti-angiogenic ability of the T7 peptide. The anti-angiogenic ability of the $\mathrm{T} 7$ peptide was partially antagonized by the autophagy induced by the $\mathrm{T} 7$ peptide. There was an increase in EC apoptosis and reduction in angiogenesis, which were accompanied by inhibition of autophagy. Therefore, the combination of angiogenic and autophagic inhibitors has a great prospect for future antitumor therapies.

In summary, the present study demonstrated that the T7 peptide partially exerts its anti-angiogenic activity by 
inhibiting tumor cell viability, migration and inducing the apoptosis of ECs. Ang2 expression is upregulated by activation of the Akt pathway and improves the invasive ability of HepG2 cells, which is inhibited by the T7 peptide. Furthermore, inhibition of autophagy enhances the antiangiogenic activity of the T7 peptide to some extent, which provides evidence for the application of an autophagic inhibitor in future antitumor therapy.

\section{Acknowledgements}

This research was supported from the National Natural Scientific Foundation of China (http://www.nsfc.gov.cn) (30972890 and 81172331).

\section{References}

1. Psyrri A, Arkadopoulos N, Vassilakopoulou M, Smyrniotis V and Dimitriadis G: Pathways and targets in hepatocellular carcinoma. Expert Rev of Anticancer Ther 12: 1347-1357, 2012.

2. El-Serag HB: Hepatocellular carcinoma. N Engl J Med 365: 1118-1127, 2011.

3. Hanahan D and Folkman J: Patterns and emerging mechanisms of the angiogenic switch during tumorigenesis. Cell 86: 353-364, 1996.

4. Almog N, Ma L, Schwager C, et al: Consensus microRNAs governing the switch of dormant tumors to the fast-growing angiogenic phenotype. PLoS One 7: e44001, 2012.

5. Welker MW and Trojan J: Anti-angiogenesis in hepatocellular carcinoma treatment: current evidence and future perspectives World J Gastroenterol 17: 3075-3081, 2011.

6. Edeline J, Boucher E, Rolland Y, et al: Comparison of tumor response by Response Evaluation Criteria in Solid Tumors (RECIST) and modified RECIST in patients treated with sorafenib for hepatocellular carcinoma. Cancer 118: 147-156, 2012.

7. Zhai B and Sun XY: Mechanisms of resistance to sorafenib and the corresponding strategies in hepatocellular carcinoma. World J Hepatol 5: 345-352, 2013.

8. Liang Y, Zheng T, Song R, et al: Hypoxia-mediated sorafenib resistance can be overcome by EF24 through Von Hippel-Lindau tumor suppressor-dependent HIF-1 $\alpha$ inhibition in hepatocellular carcinoma. Hepatology 57: 1847-1857, 2013.

9. Paez-Ribes M, Allen E, Hudock J, et al: Antiangiogenic therapy elicits malignant progression of tumors to increased local invasion and distant metastasis. Cancer Cell 15: 220-231, 2009.

10. Miyahara K, Nouso K, Tomoda T, et al: Predicting the treatment effect of sorafenib using serum angiogenesis markers in patients with hepatocellular carcinoma. J Gastroenterol Hepatol 26: 1604-1611, 2011.

11. Holash J, Maisonpierre PC, Compton D, et al: Vessel cooption, regression and growth in tumors mediated by angiopoietins and VEGF. Science 284: 1994-1998, 1999.

12. Augustin HG, Koh GY, Thurston G and Alitalo K: Control of vascular morphogenesis and homeostasis through the angiopoietin-Tie system. Nat Rev Mol Cell Biol 10: 165-177, 2009.

13. Saharinen P, Bry M and Alitalo K: How do angiopoietins Tie in with vascular endothelial growth factors? Cur Opin Hematol 17: 198-205, 2010.

14. Boosani CS, Mannam AP, Cosgrove D, et al: Regulation of COX-2 mediated signaling by alpha3 type IV noncollagenous domain in tumor angiogenesis. Blood 110: 1168-1177, 2007.

15. Wang W, Xu CX, Hou GS, Chen YG, Xin JX and Liu XX: Downregulation of tumstatin expression by overexpression of ornithine decarboxylase. Oncol Rep 30: 2042-2048, 2013.

16. Colorado PC, Torre A, Kamphaus G, et al: Anti-angiogenic cues from vascular basement membrane collagen. Cancer Res 60: 2520-2526, 2000.

17. Maeshima Y, Sudhakar A, Lively JC, et al: Tumstatin, an endothelial cell-specific inhibitor of protein synthesis. Science 295: 140-143, 2002.
18. Yamamoto Y, Maeshima Y, Kitayama H, et al: Tumstatin peptide, an inhibitor of angiogenesis, prevents glomerular hypertrophy in the early stage of diabetic nephropathy. Diabetes 53: 1831-1840, 2004.

19. Glick D, Barth S and Macleod KF: Autophagy: cellular and molecular mechanisms. J Pathol 221: 3-12, 2010.

20. Zhu H, Wang D, Zhang L, et al: Upregulation of autophagy by hypoxia-inducible factor- $1 \alpha$ promotes EMT and metastatic ability of $\mathrm{CD} 133^{+}$pancreatic cancer stem-like cells during intermittent hypoxia. Oncol Rep 32: 935-942, 2014.

21. Dong X, Li R, Xiu P, et al.: Meloxicam executes its antitumor effects against hepatocellular carcinoma in COX-2-dependent and -independent pathways. PLoS One 9: e92864, 2014.

22. Roy A and Kolattukudy PE: Monocyte chemotactic proteininduced protein (MCPIP) promotes inflammatory angiogenesis via sequential induction of oxidative stress, endoplasmic reticulum stress and autophagy. Cell Signal 24: 2123-2131, 2012.

23. Sachdev U, Cui X, Hong G, et al: High mobility group box 1 promotes endothelial cell angiogenic behavior in vitro and improves muscle perfusion in vivo in response to ischemic injury. J Vasc Surg 55: 180-191, 2012.

24. Nguyen TM, Subramanian IV, Kelekar A and Ramakrishnan S: Kringle 5 of human plasminogen, an angiogenesis inhibitor, induces both autophagy and apoptotic death in endothelial cells. Blood 109: 4793-4802, 2007.

25. Hu B, Jarzynka MJ, Guo P, Imanishi Y, Schlaepfer DD and Cheng SY: Angiopoietin 2 induces glioma cell invasion by stimulating matrix metalloprotease 2 expression through the alphavbetal integrin and focal adhesion kinase signaling pathway. Cancer Res 66: 775-783, 2006.

26. Fagiani $\mathrm{E}$ and Christofori G: Angiopoietins in angiogenesis. Cancer Lett 328: 18-26, 2013.

27. Felcht M, Luck R, Schering A, et al: Angiopoietin-2 differentially regulates angiogenesis through TIE2 and integrin signaling. J Clin Invest 122: 1991-2005, 2012.

28. Kim KW, Paul P, Qiao J and Chung DH: Autophagy mediates paracrine regulation of vascular endothelial cells. Lab Invest 93: 639-645, 2013.

29. Kimura S, Fujita N, Noda T and Yoshimori T: Monitoring autophagy in mammalian cultured cells through the dynamics of LC3. Methods Enzymol 452: 1-12, 2009.

30. Carmeliet P and Jain RK: Angiogenesis in cancer and other diseases. Nature 407: 249-257, 2000

31. Eikesdal HP, Sugimoto H, Birrane G, et al: Identification of amino acids essential for the antiangiogenic activity of tumstatin and its use in combination antitumor activity. Proc Natl Acad Sci USA 105: 15040-15045, 2008.

32. Giannotta M, Trani M and Dejana E: VE-cadherin and endothelial adherens junctions: active guardians of vascular integrity. Dev Cell 26: 441-454, 2013.

33. Thomas $\mathrm{M}$ and Augustin HG: The role of the angiopoietins in vascular morphogenesis. Angiogenesis 12: 125-137, 2009.

34. Mazzieri R, Pucci F, Moi D, et al: Targeting the ANG2/TIE2 axis inhibits tumor growth and metastasis by impairing angiogenesis and disabling rebounds of proangiogenic myeloid cells. Cancer Cell 19: 512-526, 2011.

35. Rini B, Szczylik C, Tannir NM, et al: AMG 386 in combination with sorafenib in patients with metastatic clear cell carcinoma of the kidney: a randomized, double-blind, placebo-controlled, phase 2 study. Cancer 118: 6152-6161, 2012.

36. Hashizume H, Falcon BL, Kuroda T, et al: Complementary actions of inhibitors of angiopoietin-2 and VEGF on tumor angiogenesis and growth. Cancer Res 70: 2213-2223, 2010.

37. Chae SS, Kamoun WS, Farrar CT, et al: Angiopoietin-2 interferes with anti-VEGFR2-induced vessel normalization and survival benefit in mice bearing gliomas. Clin Cancer Res 16: 3618-3627, 2010.

38. Wendel HG, De Stanchina E, Fridman JS, et al: Survival signalling by Akt and eIF4E in oncogenesis and cancer therapy. Nature 428: 332-337, 2004.

39. Holopainen T, Saharinen P, D'Amico G, et al: Effects of angiopoietin-2-blocking antibody on endothelial cell-cell junctions and lung metastasis. J Natl Cancer Inst 104: 461-475, 2012.

40. Levine B and Kroemer G: Autophagy in the pathogenesis of disease. Cell 132: 27-42, 2008. 